\title{
IDETC2021-71778
}

\section{DESIGN OF A PLANAR CABLE-DRIVEN PARALLEL CRANE WITHOUT PARASITIC TILT}

\author{
Lionel Etienne ${ }^{1,2}$, Philippe Cardou ${ }^{3}$, Marceau Métillon ${ }^{1,2}$, Stéphane Caro ${ }^{1,2 *}$ \\ ${ }^{1}$ Laboratoire des Sciences du Numérique de Nantes (LS2N), UMR CNRS 6004, 44300 Nantes, France \\ ${ }^{2}$ Centre National de la Recherche Scientifique (CNRS), 44321 Nantes, France \\ ${ }^{3}$ Laboratoire de robotique, Département de génie mécanique, Université Laval, Québec, QC, Canada \\ Emails: lionel.etienne@sigma-clermont.fr, marceau.metillon@ls2n.fr \\ pcardou@gmc.ulaval.ca, stephane.caro@ls2n.fr
}

\begin{abstract}
Cable-Driven Parallel Robots (CDPRs) offer high payload capacities, large translational workspace and high dynamics performances. Their rotational workspace is generally far more limited, however, which can be resolved by using cable loops, as was shown in previous research. In the case of fullyconstrained CDPRs, cable loops can induce unwanted torques on the moving-platform, causing it to tilt and move away from its intended position, which we call parasitic tilt. Hence, the orientation accuracy of such robots is usually limited. This paper deals with the design, modelling and prototyping of a planar CDPR with infinite rotations, without parasitic tilt and without an additional motor. This robot, which we call a CableDriven Parallel Crane (CDPC), is composed of a mobile platform (MP) with an embedded mechanism and a transmission module. The MP is linked with the frame by a parallelogram of three cables to constrain its orientation, including a cable loop, as well as a fourth cable. The two-degree-of-freedom (dof) motions of the moving-platform of the CDPC and the internal dof of its embedded mechanism are actuated by a total of three actuators, which are fixed to the frame. As a consequence, the overall system is fully-actuated, its total mass and inertia in motion is reduced and it is free of parasitic tilts.
\end{abstract}

\section{INTRODUCTION}

CDPRs belong to a particular class of parallel robots where a moving-platform (MP) is linked to a base frame using cables. Motors are mounted on a rigid base frame and drive winches. The cables coiled on these winches are routed through exit points located on the rigid frame to anchor points on the moving-platform. The MP pose (position and orientation) is determined by controlling the cable lengths. CDPRs hold several advantages over classical parallel robots. They are inexpensive and can cover large workspaces [1]. The lightweight cables contribute to the lower inertia of the moving-platform and consequently to a better dynamic performance over classical parallel robots [2]. Another characteristic of CDPRs is their reconfigurability. Changing the overall geometry of the robot can be done by changing the positions of its exit points and anchor points. Reconfigurability of the CDPRs is suitable for versatile applications especially in an industrial context $[3,4,5]$. CDPRs have drawn researchers' interests towards robotic applications such as pick-and-place operations, robotic machining, manipulation, intralogistics measurements and calibration systems [6, 7, 8, 9]. Cable-Driven Parallel Cranes (CDPCs) can offer extremely large two-degree of freedom translational workspace. One cannot write the same of their rotational workspace, which is generally limited both by cable interferences [10,11] and by bounds on their tensions. Several researchers have proposed solutions to this problem. Pott and Miermeister [12,13] have proposed to resolve this problem by replacing a rigid moving-platform with an articulated dyad driven through nine or even twelve cables and by as many motors. Fortin-Côté et al. [14], also opted for replacing the MP with an articulated dyad in order to generate unlimited rotations, but they resorted to a cable loop circulating on an embedded drum to drive this added rotation. This allowed them to reduce the number of cables and motors to seven, thereby

*Address all correspondence to this author. 
reducing cost, complexity and the likelihood of interference. Liu et al. [15] and Khakpour et al. [16, 17, 18] had previously developed the idea of using cable loops in CDPRs, but for the purpose of extending their translational workspaces, not their rotational workspaces. Pursuing large rotational workspaces while keeping the number of motors and cables to a minimum, Lessanibahri et al. [19] proposed a simple fully-constrained CDPR crane with three degrees of freedom. This design uses as many motors and cables as it had degrees of freedom, making it simpler than those reported by either Pott and Miermeister [13] or Fortin-Côté et al. [14]. It comes with one important drawback, however: any torque applied through the cable loop to the embedded pulley driving the dyad tilts the movingplatform. This drawback is due to the fully-constrained nature of this CDPR, by which it relies on gravity to keep its posture. In contrast, the overconstrained designs $[13,14]$ are not susceptible to this problem.

In this paper, we aim at fixing this problem, i.e., at making the moving-platform orientation independent from the torques applied by the cable loop while using no more motors than the number of degrees of freedom of the CDPR. To this end, we present the concept of a planar CDPC composed of a MP with an embedded mechanism and a transmission module, all of which are described in Section 2. The MP is linked to the frame through a parallelogram architecture made up of three cables, which constrain its orientation, and include a cable-loop to actuate its embedded mechanism. A fourth cable is used to connect the MP to the frame and obtain a large translational workspace. The design and manufacturing of a transmission system is also described. Its aim is to control both the parallelogram elongation and the cable-loop circulation, the latter being used to actuate the embedded mechanism onto the MP. This solution provides the robotic system with three inputs (three motors) and three outputs (three dof). The mathematical relationships between these inputs and outputs are derived in Section 3. A prototype of the robot and an experimental validation are presented in Section 4. The results from these trials are discussed in Section 5 and conclusions are drawn in Section 6. Let us begin with a description of the proposed robot in the following section.

\section{ROBOT ARCHITECTURE}

The photograph of Fig. 1 presents the proposed manipulator. Its architecture consists of a MP with an embedded mechanism actuated by a four-cable parallelogram architecture, which includes a cable loop. Thanks to the parallelogram architecture [20], which is noticeable in Fig. 1, the rotations of the MP in the working plane are constrained. The transmission module is located in the lower-left corner of Fig. 1, the cable exit points structure appear in the upper-left corner of the Frame.

Figure 2 shows a simplified schematic of the proposed robot. The actuation system encompasses the left part of the mechanism, which includes drums 1, 2 and 3 driven by motors $\mathrm{A}$ and $\mathrm{B}$ through a transmission mechanism, and the right part,

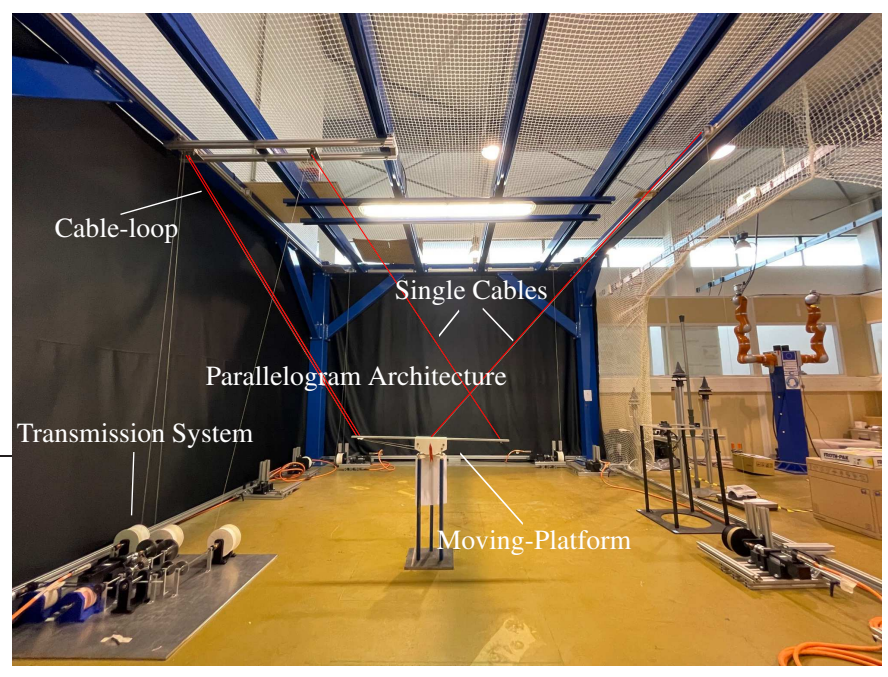

Figure 1: CDPC with parallelogram architecture and cable-loop

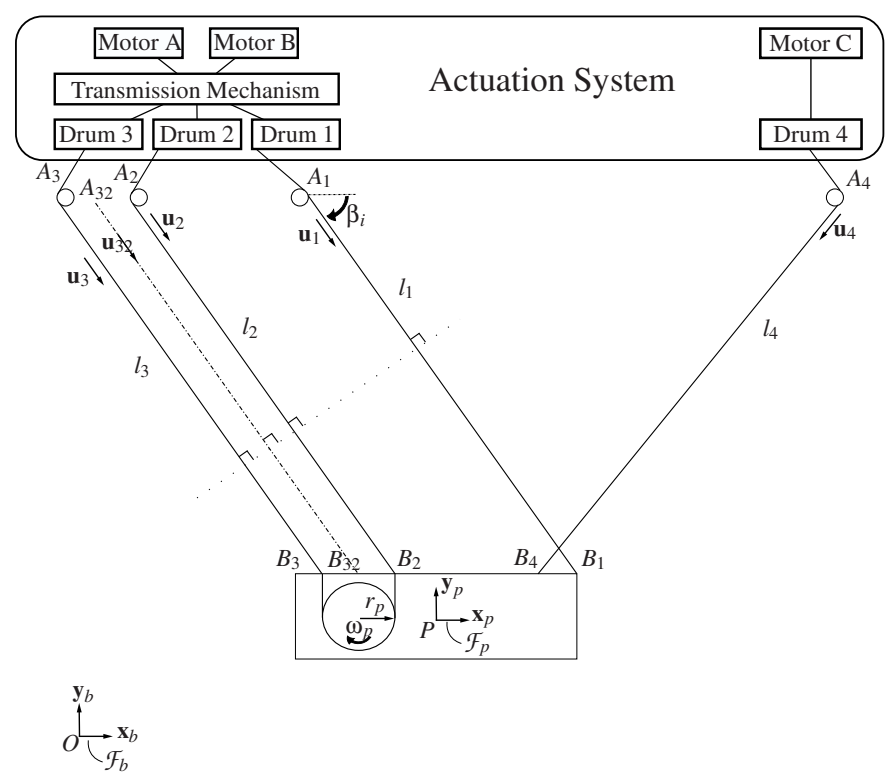

Figure 2: Simplified concept scheme of the overall mechanism

which includes drum 4 directly driven by motor $\mathrm{C}$. Attached to the frame ceiling, the exit points $A_{1}, A_{2}, A_{3}$ and $A_{4}$ guide the four cables to the anchor points $B_{1}, B_{2}, B_{3}$ and $B_{4}$. The distances between the exit point $A_{i}$ and the anchor point $B_{i}$ is the cable length $l_{i}, i=1, \ldots, 4$. The unit vectors $\mathbf{u}_{i}, i=1, \ldots, 4$, are the directions of the corresponding cables.

The cable-loop parallelogram $A_{2} A_{3} B_{3} B_{2}$ drives a pulley of radius $r_{p}$ and can be modelled as a single cable. We call this cable $A_{32} B_{32}$, its direction being unit vector $\mathbf{u}_{32}$ and its length, by $l_{32}$. The distances between $A_{1}$ and $A_{32}$ and $B_{1}$ and $B_{32}$ must be the same to maintain an overall parallelogram architecture $A_{1} A_{32} B_{32} B_{1} . \mathcal{F}_{b}$ is the fixed frame and $\mathcal{F}_{p}$ is the frame attached to the MP. 


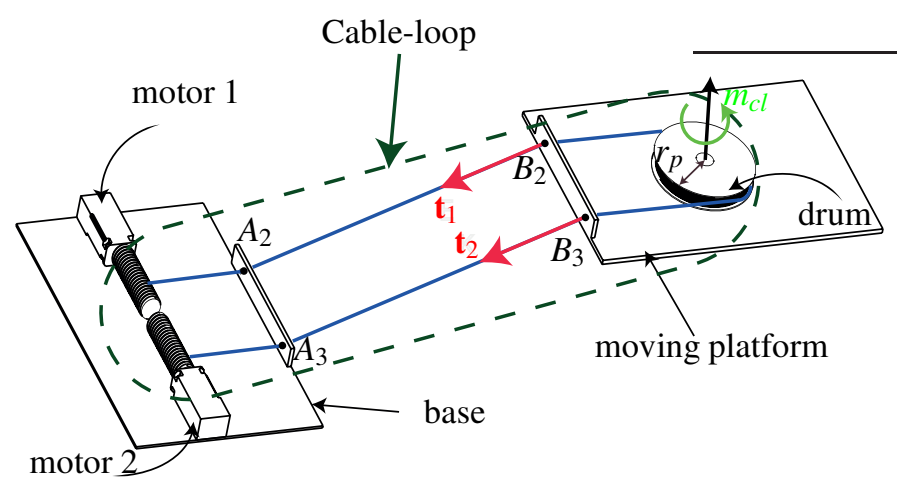

Figure 3: Representation of a cable-loop [23]

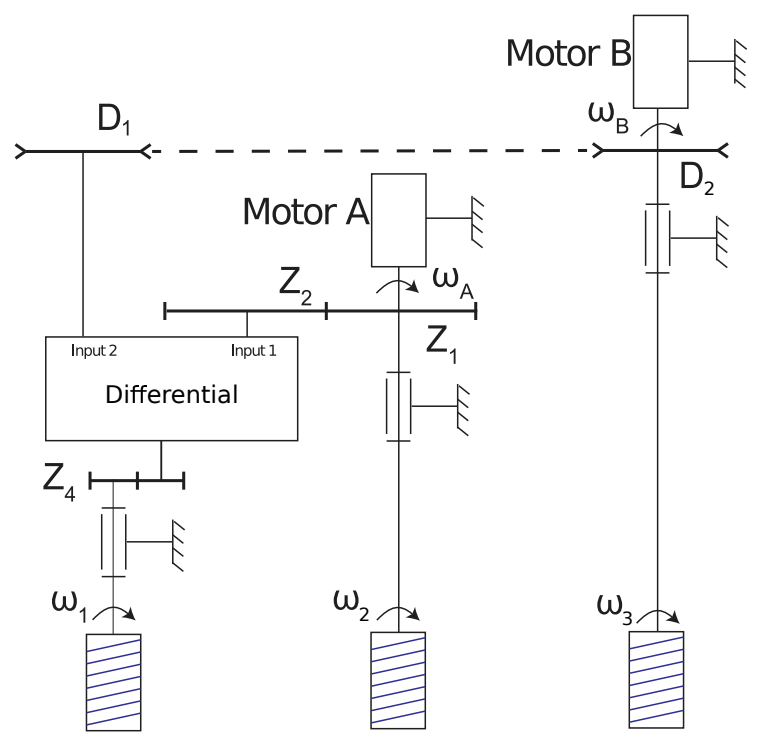

Figure 4: Transmission system scheme

\subsection{CABLE-LOOP PRINCIPLE}

As explained in [21,22] and shown in Fig. 3, the cable-loop consists of a single cable. Both of its ends are coiled on separate drums, which in turn, are actuated by two motors. Eyelets are used to maintain constant exit-points, namely, $A_{2}, A_{3}$, and anchor-points, namely, $B_{2}, B_{3}$. The cable-loop is coiled around a drum on the moving-platform. This drum then acquires one rotational DoF with respect to the moving-platform, which is used either to actuate a tool or to control additional degrees of freedom such as rotations over wide ranges. The purpose of the cable-loop is twofold. First, it aims at translating the moving platform when the coiling speeds of both motors have the same magnitude and direction. Second, it aims at rotating the embedded drum by circulating the cable when both motors have the same coiling speeds, but in opposite directions.

\subsection{TRANSMISSION SYSTEM}

The transmission system is described in Fig. 4. To control the added cable without an additional motor, the new cable

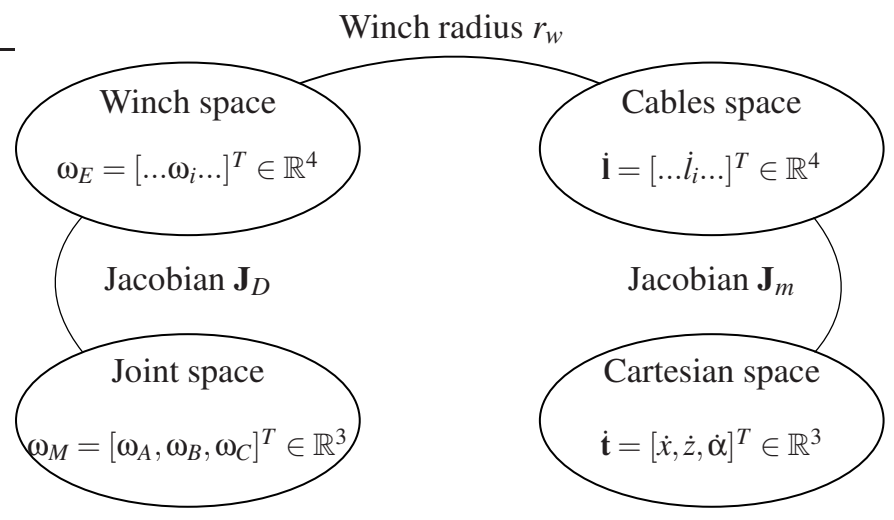

Figure 5: Joint, wrench, cable and Cartesian velocity spaces of the robot

has to follow the cable-loop elongation without being affected by its circulation. The solution proposed in this article is to use a differential mechanism knowing that the coiling speed of the added cable-here, cable 1 - is then the mean of those of cables 2 and 3.

The two motors A and B directly drive the winches 2 and 3 , and indirectly the winch 1 using a differential mechanism. The motor A actuates the latter through a gear and the motor B through a timing belt. The output of the differential drives the shaft 1 through a belt, in order to make the distance between them modular, and a gear to invert the direction of rotation.

The rotation velocities of the three motors $\omega_{A} \omega_{B}$ and $\omega_{C}$ are defined in vector $\omega_{\mathbf{M}}$. The rotation velocities of the drums $\omega_{1}, \omega_{2}, \omega_{3}$ and $\omega_{4}$ are defined in vector $\omega_{\mathbf{E}}$. All the gear and timing pulley ratios of the transmission system are 1:1.

\subsection{PARAMETERISATION}

Robot modelling is segmented into four spaces : Cartesian space, cable-length space, winch space and joint space, as shown in Fig. 5. $\theta_{A}, \theta_{B}$ and $\theta_{C}$ are the motor position angles, $\theta_{i}, i=1, \ldots, 4$, are the winch position angles, $l_{i}, i=1, \ldots, 4$, are the cable lengths, and $x, z, \phi, \alpha$ are the Cartesian parameters. More specifically, $x$ and $z$ are the absolute coordinates of the moving-platform, $\alpha$ is the cable-loop drum position angle, and $\phi$ is the tilt angle of the moving-platform. In this paper, the parallelogram guarantees $\phi=0^{\circ}$ under the assumption that the cables are inextensible and always in tension. Having qualitatively described the functioning of the proposed CDPC, let us turn our attention to the mathematical equations representing its behavior in quasi-static conditions.

\section{KINETOSTATIC MODELLING}

This section presents the kinetostatic modelling of the robot. In order to cover all the spaces defined in Subsection 2.3, let us start from the joint space. Thence, we can move successively to the winch space, the cable space and the Cartesian space. 


\subsection{Transmission Modelling}

The transmission system is the link between the joint space and the winch space, as shown in Fig. 5. The arrays of motor and winch angular velocities are respectively called $\omega_{M}$ and $\omega_{E}$, i.e.,

$$
\omega_{M}=\left[\begin{array}{l}
\omega_{A} \\
\omega_{B} \\
\omega_{C}
\end{array}\right] \text { and } \omega_{E}=\left[\begin{array}{l}
\omega_{1} \\
\omega_{2} \\
\omega_{3} \\
\omega_{4}
\end{array}\right]
$$

The motors $\mathrm{A}, \mathrm{B}$ and $\mathrm{C}$ directly drive the winches 2,3 and 4 ,

$$
\begin{aligned}
& \omega_{2}=\omega_{A} \\
& \omega_{3}=\omega_{B} \\
& \omega_{4}=\omega_{C}
\end{aligned}
$$

Because motors A and B are linked to drum 1 through the differential, their angular velocities are related through the equation,

$$
\omega_{1}=\frac{\omega_{A}+\omega_{B}}{2}=\frac{\omega_{2}+\omega_{3}}{2} .
$$

Indeed, as presented in section 2.2, the equation relating the input and output angular velocities of a differential, reproduced below in equation (6), is a mean of the input angular velocities.

$$
\omega_{\text {Output }}=\frac{\omega_{\text {Input } 1}+\omega_{\text {Input } 2}}{2}
$$

In summary, the equations (7) and (8) describe the Jacobian $\mathbf{J}_{D}$, which maps the winch angular velocities $\omega_{E}$ onto the motor angular velocities $\omega_{M}$ :

$$
\begin{gathered}
\mathbf{J}_{D}=\left[\begin{array}{ccc}
\frac{1}{2} & \frac{1}{2} & 0 \\
1 & 0 & 0 \\
0 & 1 & 0 \\
0 & 0 & 1
\end{array}\right], \\
\omega_{E}=\mathbf{J}_{D} \omega_{M} .
\end{gathered}
$$

It is a simple matter to note that the uncoiling speed $\dot{l}_{i}$ of the $i^{\text {th }}$ cable is related to the corresponding drum angular velocity $\omega_{i}$ through the equation $\dot{l}_{i}=r_{w} \omega_{i}$. Upon combining this relation with equation (8), one obtains a mapping between motor speeds and cable uncoiling speeds. Extending this relation to the moving-platform Cartesian displacements is the topic of the following subsection.

\subsection{Cable-Driven Parallel Robot}

From the parametrisation of the CDPC shown in Fig. 2 recall that both $A_{1} B_{1} B_{2} A_{2}$ and $A_{1} B_{1} B_{3} A_{3}$ are parallelograms. Also, let us define the position of point $A_{i}$ expressed in frame $\mathcal{F}_{b}$ as ${ }^{b} \mathbf{a}_{i}$, the position of point $B_{i}$ in frame $\mathcal{F}_{p}$ as ${ }^{p} \mathbf{b}_{i}$, and the rotation matrix taking a vector from frame $\mathcal{F}_{b}$ to frame $\mathcal{F}_{p}$ as ${ }^{b} \mathbf{R}_{p}$.

3.2.1 Instantaneous Kinematics The first step towards the kinematic modelling of the manipulator is to write its loop closure equations, i.e,

$$
\overrightarrow{A_{i} B_{i}}=l_{i} \mathbf{u}_{i}=\overrightarrow{A_{i} O}+\overrightarrow{O P}+\overrightarrow{P B_{i}}=-{ }^{b} \mathbf{a}_{i}+{ }^{b} \mathbf{p}+{ }^{b} \mathbf{R}_{p}{ }^{p} \mathbf{b}_{i}
$$

with $i=1,2,3,4$.

The cable loop $A_{3} B_{3} B_{2} A_{2}$ is used to control both the length of the virtual cable $A_{32} B_{32}$ and the rotations of the embedded mechanism. By differentiation of Eq. (9), we obtain

$$
\begin{array}{r}
l_{i} \mathbf{u}_{i}+l_{i} \frac{d \mathbf{u}_{i}}{d t}={ }^{\dot{p}} \dot{\mathbf{p}}+\frac{d^{b} \mathbf{b}_{i}}{d t}-\frac{d^{b} \mathbf{a}_{i}}{d t}, \\
\dot{l}_{i} \mathbf{u}_{i}+l_{i} \dot{\beta}_{i} \mathbf{E} \mathbf{u}_{i}={ }^{b} \mathbf{p}+\dot{\varphi} \mathbf{E}^{b} \mathbf{b}_{i}
\end{array}
$$

where $d^{b} \mathbf{a}_{i} / d t=0$ because $\mathbf{a}_{i}$ is constant in $\mathcal{F}_{b}$,

$$
\mathbf{E}=\left[\begin{array}{cc}
0 & -1 \\
1 & 0
\end{array}\right] \text { and }{ }^{b} \mathbf{b}_{i}={ }^{b} \mathbf{R}_{p}{ }^{p} \mathbf{b}_{i}
$$

With $\varphi=0^{\circ}$, the orientation of the MP,

$$
\dot{l}_{i} \mathbf{u}_{i}+l_{i} \dot{\beta_{i}} \mathbf{E} \mathbf{u}_{i}={ }^{b} \dot{\mathbf{p}}+\dot{\varphi} \mathbf{E}^{b} \mathbf{R}_{p}{ }^{p} \mathbf{b}_{i}
$$

where $\dot{\beta}_{i}$ is the angular velocity of cable $i$ in the fixed frame $\mathcal{F}_{b}$. In order to eliminate this passive variable, we multiply the Eq. (13) by $\mathbf{u}_{i}^{T}$ :

$$
\dot{l}_{i} \mathbf{u}_{i}^{T} \mathbf{u}_{i}+l_{i} \dot{\alpha}_{i} \mathbf{u}_{i}^{T} \mathbf{E} \mathbf{u}_{i}=\mathbf{u}_{i}^{T} \dot{\mathbf{b}} \mathbf{p}+\dot{\varphi} \mathbf{u}_{i}^{T} \mathbf{E}^{b} \mathbf{R}_{p}{ }^{p} \mathbf{b}_{i}
$$

The result can be written in matrix form :

$$
\mathbf{A} \mathbf{t}=\mathbf{B} \mathbf{i}
$$

where

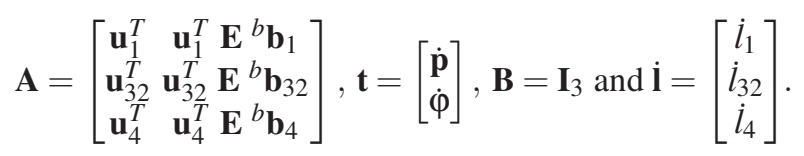


Assuming that the embedded drum is composed of a pulley of radius $r_{p}$, we obtain

$$
\omega_{p}=\frac{l_{3}-\dot{l}_{2}}{2 r_{p}} .
$$

This leads to the overall Jacobian matrices :

$$
\mathbf{A}_{g} \mathbf{t}_{g}=\mathbf{B}_{g} \dot{\mathbf{I}}_{g}
$$

with,

$$
\begin{aligned}
& \mathbf{A}_{g}=\left[\begin{array}{cccc}
\mathbf{u}_{1}^{T} & \mathbf{u}_{1}^{T} & \mathbf{E}^{b} \mathbf{b}_{1} & 0 \\
\mathbf{u}_{32}^{T} & \mathbf{u}_{32}^{T} & \mathbf{E}^{b} \mathbf{b}_{32} & 0 \\
\mathbf{u}_{4}^{T} & \mathbf{u}_{4}^{T} & \mathbf{E}^{b} \mathbf{b}_{4} & 0 \\
0 & 0 & 1
\end{array}\right], \mathbf{t}_{g}=\left[\begin{array}{c}
\dot{\mathbf{p}} \\
\dot{\varphi}
\end{array}\right], \\
& \mathbf{B}_{g}=\left[\begin{array}{cccc}
1 & 0 & 0 & 0 \\
0 & \frac{1}{2} & \frac{1}{2} & 0 \\
0 & 0 & 0 & 1 \\
0 & -\frac{1}{2 r_{p}} & \frac{1}{2 r_{p}} & 0
\end{array}\right], \quad \mathbf{i}_{g}=\left[\begin{array}{l}
\dot{l}_{1} \\
\dot{l}_{2} \\
\dot{l}_{3} \\
\dot{l}_{4}
\end{array}\right] \text {, }
\end{aligned}
$$

where $\mathbf{A}_{g} \in \mathbb{R}^{4 \times 4}$ and $\mathbf{B}_{g} \in \mathbb{R}^{4 \times 4}$. Thus, if one needs to compute the cable uncoiling speeds from a prescribed twist of the end-effector, he or she can readily solve equation (17) as $\mathbf{i}=\mathbf{J}_{m} \mathbf{t}$, where $\mathbf{J}_{m}=\mathbf{B}_{g}^{-1} \mathbf{A}_{g}$.

3.2.2 Static equilibrium From the the application of the principle of virtual work to equation (17), we obtain

$$
\mathbf{W} \tau+\mathbf{w}_{\text {ext }}=\mathbf{0}_{4}
$$

with

$$
\mathbf{W}=-\mathbf{J}_{m}^{T}=-\mathbf{A}_{g}^{T} \mathbf{B}_{g}^{-T},
$$

where $\mathbf{W} \in \mathbb{R}^{4 \times 4}$ is the wrench matrix of the manipulator, $\tau=$ $\left[\begin{array}{llll}\tau_{1} & \cdots & \tau_{4}\end{array}\right]^{T}$ is the array of cable tensions, and $\mathbf{w}_{\text {ext }}=\left[\begin{array}{ll}0 & -\end{array}\right.$ $\left.m g \quad 0 m_{E}\right]^{T}$ is the external wrench applied on the end effector. The cable tension vector $\tau$ can be obtained from the inverse of the wrench matrix $\mathbf{W}$ as long as it is not rank-deficient, namely, $\operatorname{det}(\mathbf{W}) \neq 0$.

$$
\tau=-\mathbf{W}^{-1} \mathbf{w}_{\text {ext }}
$$

From equation (8) and the principle of virtual work, the motor torques can be obtained as functions of the cable tensions. Let $\gamma_{M}=\left[\gamma_{A} \gamma_{B}\right]^{T}$ be the two-dimensional vector containing the torques applied by the two motors of the transmission and $\tau_{E}=\left[\begin{array}{lll}\tau_{1} & \tau_{2} & \tau_{3}\end{array}\right]^{T}$, the three-dimensional vector containing the moments applied by the transmission to the three drums 1 , 2 and 3. From the principle of virtual work, the input work amounts to the output work as long as there is no loss in the transmission system. As a consequence,

$$
\begin{aligned}
\gamma_{M}^{T} \omega_{M} & =\tau_{E}^{T} \omega_{E} \\
\gamma_{M}^{T} \omega_{M} & =\tau_{E}^{T} \mathbf{J}_{D} \omega_{M}
\end{aligned}
$$

Finally, the motor torques are expressed as a function of the moments exerted on the first three drums as follows:

$$
\gamma_{M}=\mathbf{J}_{D}^{T} \tau_{E}
$$

3.2.3 Inverse Kinematics of the Transmission System $\theta_{1}(t), \theta_{2}(t), \theta_{3}(t)$ are the position angles of the winches at time $t$ in $\operatorname{rad}$ and $\theta_{A}(t) \theta_{B}(t)$ are the position angles of the output shaft of system \{motor+ gearhead $\}$ at time $t$ in rad, we can readily find :

$$
\begin{aligned}
& \theta_{1}(t)=\frac{1}{2}\left[\left(\theta_{A}(t)-\theta_{A}(0)\right)+\left(\theta_{B}(t)-\theta_{B}(0)\right)\right]+C \\
& \theta_{2}(t)=\theta_{A}(t)-\theta_{A}(0) \\
& \theta_{3}(t)=\theta_{B}(t)-\theta_{B}(0)
\end{aligned}
$$

We can assume that $\theta_{A}(0)=\theta_{B}(0)=0$, which yields $C=\theta_{1}(0)$. With the mathematical relationships derived in this section, we are equipped to control the motion of the proposed robot, which allows us to move to its experimental validation.

\section{PROTOTYPING AND EXPERIMENTATION}

In this section, we present an embodiment of the proposed concept and two test trajectories: one where pure translations and pure rotations are performed in sequence, and the other where they are combined in a generic motion.

\subsection{Prototype presentation}

The experimental demonstrator of the manipulator is manufactured as shown in Figs. 1 and 6. The architecture of the CDPC is presented in Fig. 9. The main hardware of the CREATOR demonstrator is shown in Fig. 7 and consists of a PC (equipped with ${ }^{C}$ MATLAB and C ControlDesk software), a controller, a motor driver, winch assemblies, each comprising a servomotor, a gearbox and a drum and a moving platform. This testbed enables us to evaluate the capabilities of the proposed manipulator in operation.

This remainder of this section explains the experimental process followed to verify the viability of the concept. In particular, we wish to verify how susceptible it is to parasitic tilts by comparison with a previous design that did not involve a 


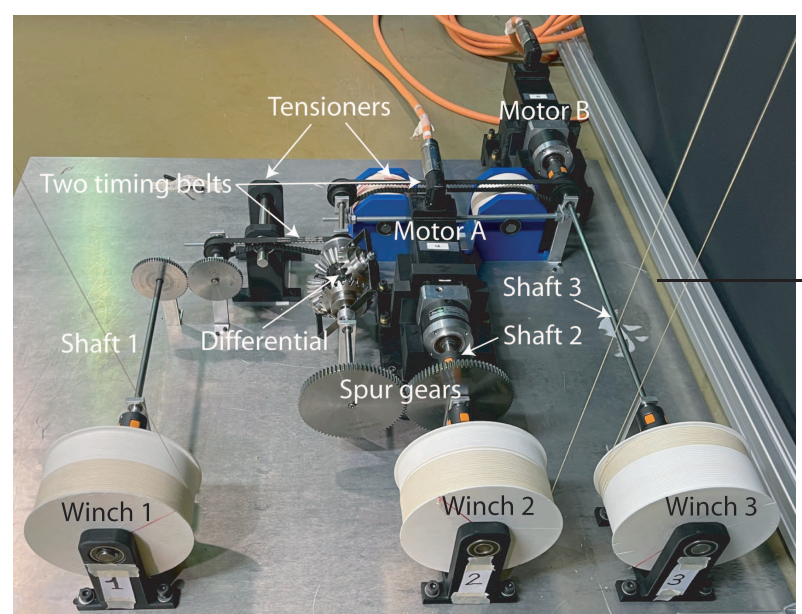

Figure 6: Photograph of the Transmission system

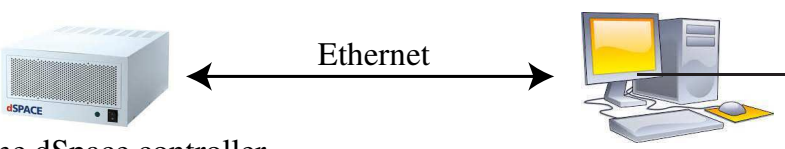

Real-time dSpace controller

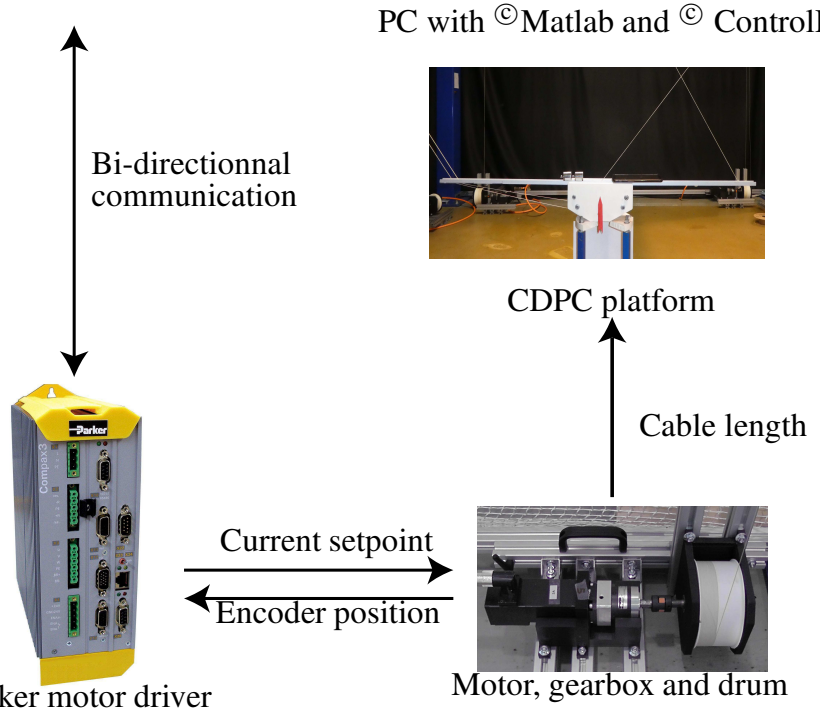

Figure 7: Control architecture of the manipulator

parallelogram. Firstly, the trajectories are defined in subsection 4.3. Then, the experiments 1 and 2 are described and discussed in sections 4.3.1 and 4.3.2, respectively.

\subsection{Configurations}

The goal of these experiments is to assess the effectiveness of the proposed architecture in preventing the parasitic tilts generated when applying moments through a cable loop. We do this by comparing the behaviour of our parallelogram configuration with that of a previous architecture without parallelogram or differential transmission. This reference configuration is presented in [19], and may be described as an underconstrained CDPR with three cables, three actuators and four

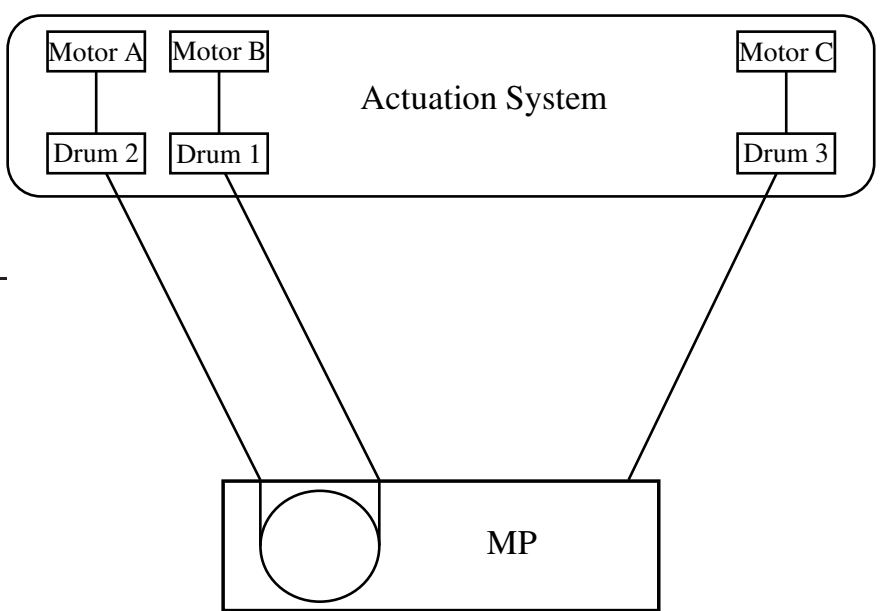

(a) Concept schematic of the three-cable configuration

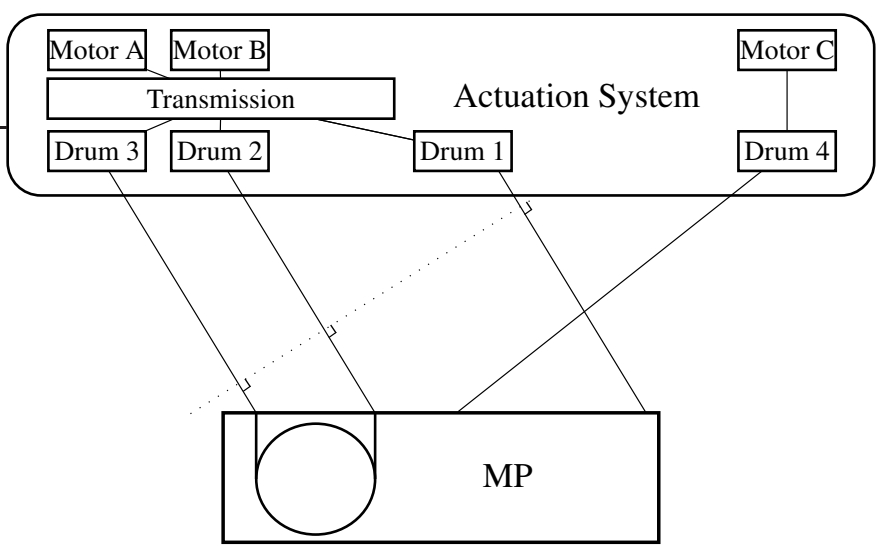

(b) Concept schematic of the four-cable configuration

Figure 8: Schematics of the two robot configurations under study

dof. We call it $\mathcal{C}_{1}$. The configuration proposed in this article is shown in Fig. 2, and is a fully-actuated configuration with four cables, three actuators and three dof. We call it $\mathcal{C}_{2}$. These two configurations are shown in Figs. 9 (a) and (b), respectively.

\subsection{Trajectories}

Two trajectories were chosen in order to evaluate the performance of the parallelogram with respect to the platform orientation. One trajectory consists in moving separately the translation and rotation DoF of the inner mechanism; The other trajectory is a combination of these two motions. A polynomial of order five was used to define the trajectories.

\subsubsection{Trajectory 1 - Pure Translations and Rota-} tions in Sequence The first trajectory, shown in Fig. 10, is a succession of translations and embedded-mechanism rotations. It may be described step by step as follows:

(a) Vertical translation from the stand $P_{0}$ to the position above the stand $P_{1}(33 \mathrm{~s} \leq t \leq 43 \mathrm{~s})$ 


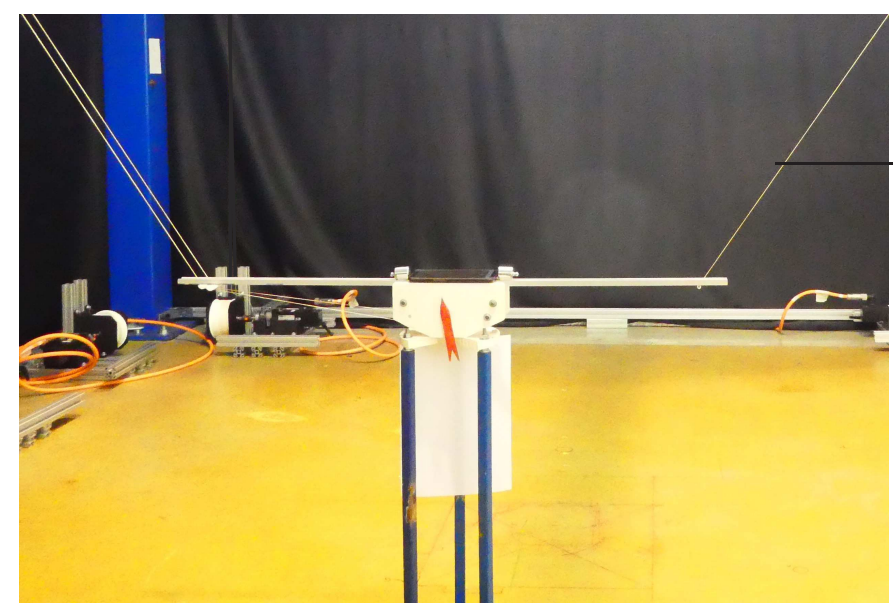

(a) Photograph of the three-cable configuration

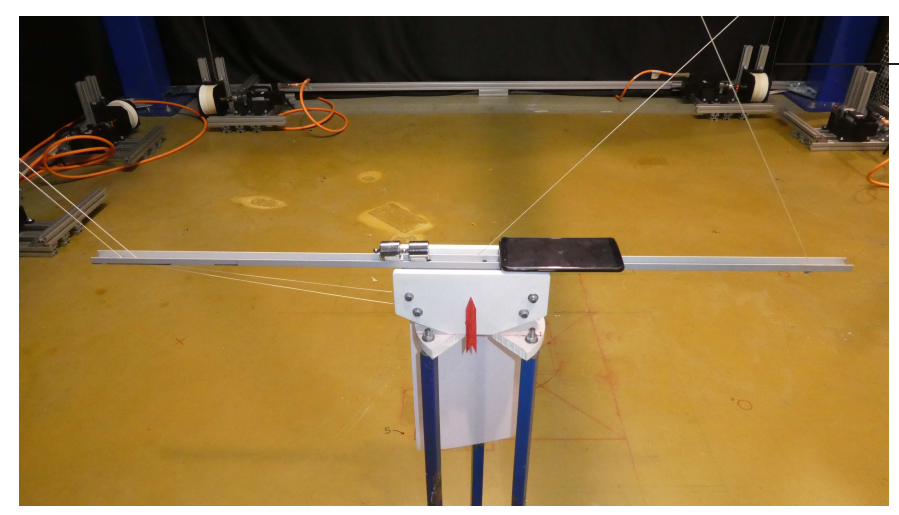

(b) Photograph of the four-cable configuration

Figure 9: The two robot configurations

(b) Diagonal translation from $P_{1}$ to $P_{2}(43 \mathrm{~s} \leq t \leq 53 \mathrm{~s})$

(c) Rotation anticlockwise of the embedded mechanism at $P_{2}$ $(55 \mathrm{~s} \leq t \leq 65 \mathrm{~s})$

(d) Rotation clockwise of the embedded mechanism at $P_{2}$ $(63 \mathrm{~s} \leq t \leq 73 \mathrm{~s})$

(e) Horizontal translation from $P_{2}$ to $P_{3}(73 \mathrm{~s} \leq t \leq 93 \mathrm{~s})$

$(f)$ Rotation anticlockwise of the embedded mechanism at $P_{3}$ $(93 \mathrm{~s} \leq t \leq 103 \mathrm{~s})$

(g) Rotation clockwise of the embedded mechanism at $P_{3}$ $(103 \mathrm{~s} \leq t \leq 113 \mathrm{~s})$

(h) Diagonal translation from $P_{3}$ to $P_{1}(113 \mathrm{~s} \leq t \leq 123 \mathrm{~s})$

(i) Vertical translation from above stand $P_{1}$ to stand position $(123 \mathrm{~s} \leq t \leq 133 \mathrm{~s})$

Point $O$ being the origin of frame $\mathcal{F}_{b}$, the Cartesian coordinates of the control points of the Trajectory 1 are $\overrightarrow{O P_{0}}=$ $\left[\begin{array}{ll}1.646 & 0.691\end{array}\right]^{T} \mathrm{~m}, \overrightarrow{O P_{1}}=\left[\begin{array}{ll}1.646 & 0.841\end{array}\right]^{T} \mathrm{~m}, \overrightarrow{O P_{2}}=\left[\begin{array}{ll}1 & 1\end{array}\right]^{T} \mathrm{~m}$, and $\overrightarrow{O P_{3}}=\left[\begin{array}{lll}2.5 & 1\end{array}\right]^{T} \mathrm{~m}$.

\subsubsection{Trajectory 2 - Combined Translations and}

Rotations The second trajectory is a combined movement of translation and cable loop rotation at the same time as

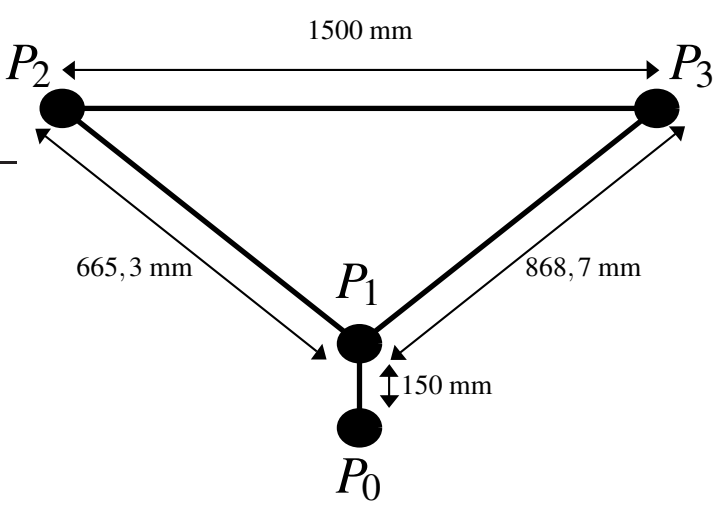

Figure 10: Path of Trajectory 1 - pure translations and rotations in sequence

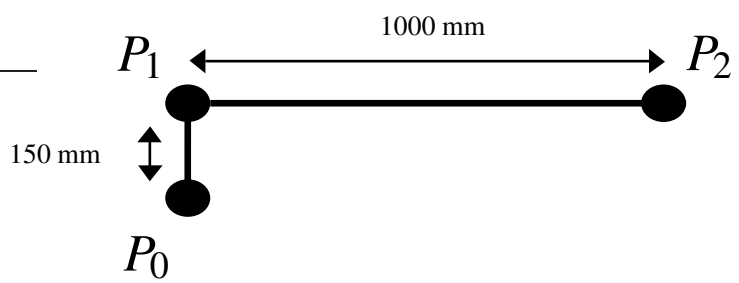

Figure 11: Path of the Trajectory 2 - combined translations and rotations

presented in Fig. 11. The steps :

(a) Vertical translation from the stand to the position $P_{1}$ above it $(15 \mathrm{~s} \leq t \leq 25 \mathrm{~s})$

(b) Rotation anticlockwise of the embedded mechanism at position $P_{1}$ above the stand $(25 \mathrm{~s} \leq t \leq 35 \mathrm{~s})$

(c) Horizontal translation while the embedded mechanism rotates clockwise from $P_{1}$ to $P_{2}(35 \mathrm{~s} \leq t \leq 65 \mathrm{~s})$

The coordinates of the Trajectory 2 control points are $\overrightarrow{O P_{1}}=[1.6460 .691]^{T} \mathrm{~m}, \overrightarrow{O P_{2}}=[2.6460 .691]^{T} \mathrm{~m}$.

\section{Result Analysis}

The experiment consists in testing the two trajectories with the three-cable configuration and then with the four-cable configuration described in Fig. 9. During the experiment illustrated in video ${ }^{1}$, the orientation around axis $z_{b}$ was measured with an inclinometer on a phone to evaluate the system ability to stabilize the platform. Counterweights were used to statically balance the platform and thus cancel the weight of the phone. Also, the inner mechanism was represented by a red arrow.

\subsection{Description of the Results - Trajectory 1}

Results of the experiments are shown in Figs. 12 and 13. The green curves describe the parasitic tilt $\phi$ of the four-cable

${ }^{1}$ video 


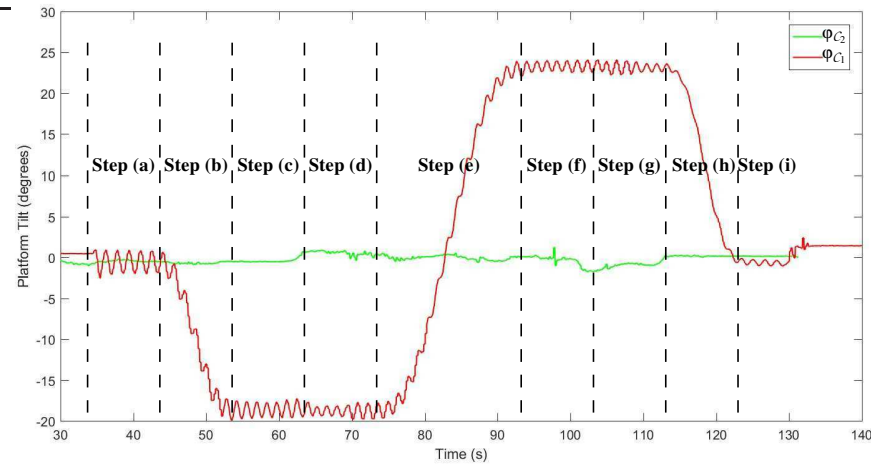

Figure 12: Tilt angle around axis $z_{b}$ - Trajectory 1

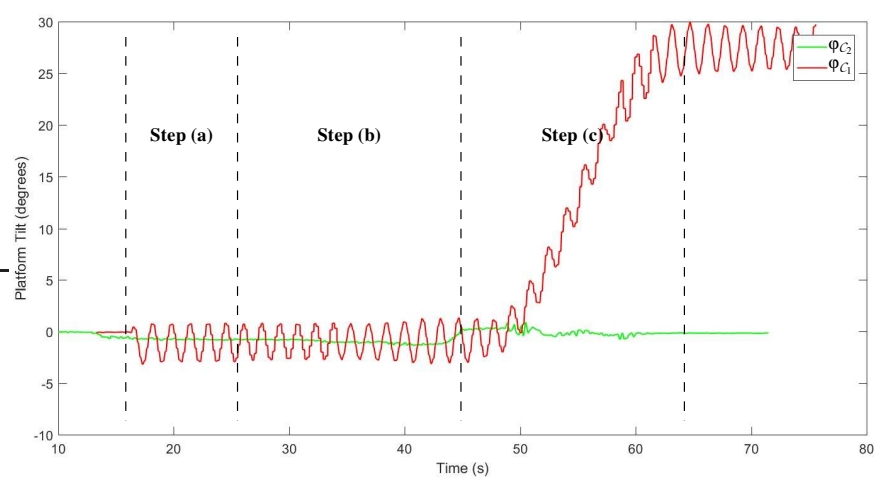

Figure 13: Tilt angle around axis $z_{b}$ - Trajectory 2

configuration angle in degrees around axis $z_{b}$ of the mobile platform. The red curves represent the same angle for the threecable configuration.

In the first trajectory, the $z_{b}$ rotation angle for the threecable configuration decreases during step $(b)$, reaches a mean of $-18^{\circ}$ during steps $(c)$ and $(d)$ increases at step $(e)$. To stabilizes around $23^{\circ}$ degrees at steps $(f)$ and $(g)$ and decreases back to $0^{\circ}$ at step $(h)$. A damped oscillation of the platform around axis $z_{b}$ is visible during the whole experiment; its period is $1.6 \mathrm{~s}$ and its total amplitude starts at around $3^{\circ}$. The $z_{b}$ rotation angle for the four-cable configuration is continuous and remains around $0^{\circ}$ degree with approximately $1^{\circ}$ of error during the whole test with no visible sign of oscillations.

\subsection{Description of the Results - Trajectory 2}

In the second trajectory, the $z_{b}$ rotation angle for the threecable configuration is continuous around $0^{\circ}$ during steps $(a)$ and $(b)$ and increases to $27^{\circ}$ during step $(c)$. As in trajectory 1 , oscillations of the moving platform can be observed by eye during the experiment. Angle measurements show that their oscillating period is $1.6 \mathrm{~s}$ and their amplitude is around $3.4^{\circ}$, figures that are closely similar to those obtained in trajectory 1 . The $z_{b}$ rotation angle for the four-cable configuration is continuous and remains around $0^{\circ}$ with approximately $1^{\circ}$ of error during the whole test with no sign of oscillations.

\subsection{Discussion and Validation of the Results}

The goal of this experiment was to demonstrate the effectiveness of the parallelogram cable structure. At the outset, we observe in both experiments that the three-cable configuration exhibits errors superior to $20^{\circ}$ consistently increasing in translation steps. We also have to consider the oscillations of the moving-platform in this configuration. Both of those errors may be attributed to the under-constrained nature of the robot. Indeed, this configuration has three motors and four DoFs, counting the translations in $\mathrm{X}$ and $\mathrm{Y}$, the rotation about $z_{b}$ and the inner DoF of the embedded mechanism.

The four-cable configuration is showing the expected results: a stable $z_{b}$ angle with approximately $1^{\circ}$ of error during the whole trajectory. Notice that a moving-platform starting with an offset angle would be bound to keep it during the entire experiment. What is important here is the continuity and stability of this angle.

The results obtained with both three- and four-cable configurations show the effectiveness of the parallelogram and validate the first hypothesis of this work. We can still observe in both trials that the $1^{\circ}$ error appears in increments at step changes. For example, error increases can be seen in trajectory 1 between steps $(c)$ and $(d),(f)$ and $(g)$ or at the end of step $(c)$. These error changes seem to come from the overall transmission play and we can confidently assert that it is in large part an inversion play that occurs when the added winch 1 changes its direction of rotation.

\subsection{Issues encountered and Solutions}

The first tests for this experiment were met with mechanical issues. The first problem was the tooth jumps of the belts when the torque applied was too high caused by the insufficient belt tensions and the differential assembly being too flexible laterally. To avoid those jumps, the solutions was to add belt tensioners, to reinforce the in-between support tops with threaded rods and to reduce the weight. Unfortunately we were unable to remove the play inside the differential caused by an overly flexible satellite carrier. Likewise, we could not remove the plays in between tooths of the conical gears and in the flexible coupling.

At some point we decided to improve the stabilization by making the parallelogram wider in order to better demonstrate the potential of our concept. Along the same line, we reduced the weight of the moving-platform by 3D-printing its frame and removing the embedded mechanism, replacing its hoist by a simple rotating arrow.

Concerning the cable loop, we can observe that the cable is slipping on the embedded mechanism during the experiment. Possible solutions would be to add weight on the platform to increase the cable tension or to make the drum surface more rough so as to increase friction.

During the tests, we also detected that the workspace was strongly impacted by the weight and the position of the 4th cable anchor point onto the mobile platform itself. It appears that positioning point $B_{4}$ in the middle of the platform, more pre- 
cisely at mid-distance between $B_{1}$ and $B_{32}$, increases significantly the workspace of the robot.

\section{Conclusion and Future Work 6.1 Conclusion}

The main challenge of the article was to remove the unwanted rotations of the mobile platform of a planar CDPR with three actuators. The proposed solution was to add a cable to form a parallelogram capable of constraining the movingplatform rotations while keeping intact the cable loop system to activate the inner embedded mechanism and the number of motors. In order to achieve this challenge we designed a transmission module composed of a differential to control the added cable with the two motors on the left side of the robot. Finally, the experiments showed the viability and effectiveness of the solution proposed, as its rotation errors reached approximately $1^{\circ}$, compared to approximately $25^{\circ}$ for the previously existing solution [19].

\subsection{Future Work}

As discussed in subsection 5.4, the first important point of improvement is to increase the stiffness of the transmission module, especially in the differential and belts. This point is vital for the accuracy of torque, position and speed of the added winch. More efficient tensioners and proper reinforcement of supports could be a solution. Furthermore, the play in-between teeth of the conical gears in the differential could be reduced by making a stiffer carrier and using helical conical gears. This application is a proof of concept and future work will consist in extending this concept to design a CDPR with a 3D workspace and capable of large rotations through cable loops.

\section{ACKNOWLEDGMENT}

This work was supported by the ANR CRAFT project, grant ANR-18-CE10-0004, https://anr.fr/Project-ANR-18-CE10-0004 and the RFI AtlanSTIC2020 CREATOR project.

\section{NOMENCLATURE}

$\mathcal{F}_{b}\left(O_{b}, x_{b}, y_{b}, z_{b}\right)$ Frame attached to the robot base $\mathcal{F}_{p}\left(O_{p}, x_{p}, y_{p}, z_{p}\right)$ Frame attached to the moving-platform

$\mathbf{1}_{i} i$-th cable vector pointing from $B_{i}$ to $A_{i}, i \in[[1, \ldots, 4]]$

$\mathbf{a}_{i} \quad$ Cartesian coordinates vector of point $A_{i}, i \in[[1, \ldots, 4]$

$\mathbf{b}_{i}$ Cartesian coordinates vector of point $B_{i}, i \in[[1, \ldots, 4]]$

p Cartesian coordinates vector of point $P$

$\mathbf{u}_{i} \quad i$-th cable unit vector, $i \in[[1, \ldots, 4]]$

t Cartesian parameter vector

$\mathbf{w}_{e}$ External wrench vector

$\tau$ Cable tension vector

$l_{i} \quad i$-th cable length, $i \in[[1, \ldots, 4]]$

$r_{p} \quad$ Radius of the embedded drum of the cable loop

$A_{i} \quad i$-th cable exit point, $i \in[[1, \ldots, 4]]$
$B_{i} \quad i$-th cable anchor point, $i \in[[1, \ldots, 4]]$

$\omega_{p} \quad$ Angular velocity of the embedded drum

$\omega_{M} \quad$ Motor angular velocity vector

$\omega_{E} \quad$ Winch angular velocity vector

$\alpha$ Rotation angle of the embedded drum

$\beta_{i} \quad$ Orientation angle of the $i$ th cable

$\varphi \quad$ Tilt angle of the moving-platform

$\tau_{i} \quad i$-th cable tension

$\gamma_{M}$ Torque applied on the moving-platform

${ }^{0} \mathbf{R}_{p} \quad$ Rotation matrix from frame $\mathcal{F}_{b}$ to frame $\mathcal{F}_{p}$

W Wrench matrix

$\mathbf{A}_{g}$ Forward Jacobian matrix of the robot

$\mathbf{B}_{g}$ Inverse Jacobian matrix of the robot

\section{REFERENCES}

[1] Hussein, H., Santos, J. C., and Gouttefarde, M., 2018. "Geometric optimization of a large scale cdpr operating on a building facade". In 2018 IEEE/RSJ International Conference on Intelligent Robots and Systems (IROS), IEEE, pp. 5117-5124. 1

[2] Kawamura, S., Choe, W., Tanaka, S., and Pandian, S. R., 1995. "Development of an ultrahigh speed robot falcon using wire drive system". In Proceedings of 1995 IEEE International Conference on Robotics and Automation, IEEE, pp. 215-220. 1

[3] Gagliardini, L., Caro, S., Gouttefarde, M., and Girin, A., 2016. "Discrete reconfiguration planning for cable-driven parallel robots". Mechanism and Machine Theory, 100, pp. 313-337. PII: S0094114X16000513. 1

[4] Rasheed, T., Long, P., and Caro, S., 2020. "Wrenchfeasible workspace of mobile cable-driven parallel robots". Journal of Mechanisms and Robotics, 12(3), Jan., p. 031009. 1

[5] Rasheed, T., Long, P., Roos, A. S., and Caro, S., 2019. "Optimization based Trajectory Planning of Mobile Cable-Driven Parallel Robots". In The 2019 IEEE/RSJ International Conference on Intelligent Robots and Systems (IROS 2019), pp. 6788-6793. 1

[6] Qian, S., Zi, B., Shang, W.-W., and Xu, Q.-S., 2018. “A review on cable-driven parallel robots". Chinese Journal of Mechanical Engineering, 31(1), p. 1007. PII: 267. 1

[7] Zake, Z., Chaumette, F., Pedemonte, N., and Caro, S., 2020. "Robust $2 d$ visual servoing of a cable-driven parallel robot thanks to trajectory tracking". IEEE Robotics and Automation Letters, 5(2), pp. 660-667. 1

[8] Bosscher, P., Williams, R. L., Bryson, L. S., and CastroLacouture, D., 2007. "Cable-suspended robotic contour crafting system". Automation in Construction, 17(1), pp. $45-55.1$

[9] Bostelman, R., Albus, J., Dagalakis, N., Jacoff, A., and Gross, J., 1994. "Applications of the nist robocrane". In Proceedings of the 5th International Symposium on Robotics and Manufacturing, Vol. 5. 1 
[10] Blanchet, L., and Merlet, J.-P., 2014. "Interference detection for cable-driven parallel robots (cdprs)". In 2014 IEEE/ASME International Conference on Advanced Intelligent Mechatronics, pp. 1413-1418. 1

[11] Bak, J.-H., Hwang, S. W., Yoon, J., Park, J. H., and Park, J.-O., 2019. "Collision-free path planning of cable-driven parallel robots in cluttered environments". Intelligent Service Robotics, 12(3), pp. 243-253. PII: 278. 1

[12] Miermeister, P., and Pott, A., 2014. "Design of cabledriven parallel robots with multiple platforms and endless rotating axes". In Interdisciplinary applications of kinematics, A. Kecskemthy and F. Geu Flores, eds., Vol. 26 of Mechanisms and Machine Science. Springer, pp. 21-29. 1

[13] Pott, A., and Miermeister, P., 2016. "Workspace and interference analysis of cable-driven parallel robots with an unlimited rotation axis". In Advances in Robot Kinematics, pp. 341-350. 1, 2

[14] Fortin-Côté, A., Faure, C., Bouyer, L., McFadyen, B. J., Mercier, C., Bonenfant, M., Laurendeau, D., Cardou, P., and Gosselin, C., 2017. "On the design of a novel cabledriven parallel robot capable of large rotation about one axis". In $3^{\text {rd }}$ International Conference on Cable-Driven Parallel Robots, Springer, pp. 390-401. 1, 2

[15] Liu, H., Gosselin, C., and Laliberté, T., 2012. "Conceptual design and static analysis of novel planar springloaded cable-loop-driven parallel mechanisms". Journal of Mechanisms and Robotics, 4(2). 2

[16] Khakpour, H., and Birglen, L., 2014. "Workspace augmentation of spatial 3-dof cable parallel robots using differential actuation". In 2014 IEEE/RSJ International Conference on Intelligent Robots and Systems, IEEE, pp. 3880-3885. 2

[17] Khakpour, H., Birglen, L., and Tahan, S.-A., 2014. "Synthesis of differentially driven planar cable parallel manipulators". IEEE Transactions on Robotics, 30(3), pp. 619630. 2

[18] Khakpour, H., Birglen, L., and Tahan, S.-A., 2015. "Analysis and optimization of a new differentially driven cable parallel robot". Journal of Mechanisms and Robotics, 7(3). 2

[19] Lessanibahri, S., Cardou, P., and Caro, S., 2018. "Kinetostatic analysis of a simple cable-driven parallel crane". In Volume 5A: 42nd Mechanisms and Robotics Conference, American Society of Mechanical Engineers. 2, 6, 9

[20] Longval, J. M., and Gosselin, C., 2019. "Dynamic trajectory planning and geometric analysis of a two-degreeof-freedom translational cable-suspended planar parallel robot using a parallelogram cable loop". Journal of Mechanisms and Robotics, 11(2). 2

[21] Lessanibahri, S., Cardou, P., and Caro, S., 2020. "A Cable-Driven Parallel Robot with an Embedded Tilt-Roll Wrist”. Journal of Mechanisms and Robotics, 12(2), Feb. 3

[22] Métillon, M., Lessanibahri, S., Cardou, P., Subrin, K., and
Caro, S., 2020. "A cable-driven parallel robot with fullcircle end-effector rotations". In Volume 10: 44th Mechanisms and Robotics Conference (MR), American Society of Mechanical Engineers. 3

[23] Métillon, M., Cardou, P., Subrin, K., Charron, C., and Caro, S., 2021. "A Cable-Driven Parallel Robot with FullCircle End-Effector Rotations". Journal of Mechanisms and Robotics, 13, June, pp. 031115-1-031115-9. 3 